\title{
A Design of Nuclear Electronics Filter Forming Circuit
}

\author{
Shaoqin Li, Yunrui Jiang, Jie Shi \\ School of Nuclear Technology \& Automation Engineering, Chengdu University of Technology, Chengdu, China \\ Email: 965035530@qq.com
}

How to cite this paper: Li, S.Q., Jiang, Y.R. and Shi, J. (2021) A Design of Nuclear Electronics Filter Forming Circuit. Open Access Library Journal, 8: e7843. https://doi.org/10.4236/oalib.1107843

Received: August 9, 2021

Accepted: August 31, 2021

Published: September 3, 2021

Copyright $\odot 2021$ by author(s) and Open Access Library Inc.

This work is licensed under the Creative Commons Attribution International License (CC BY 4.0).

http://creativecommons.org/licenses/by/4.0/

\section{(c) (i) Open Access}

\begin{abstract}
Multisim is a Windows-based simulation tool launched by National Instruments Inc., which is suitable for the design of analog/digital circuit boards at board level. It includes the graphic input of circuit schematic diagram and the input of circuit hardware description language. It has a rich simulation and analysis ability. Compared with the traditional manual calculation and design circuit, it can be designed and simulated more simply and quickly through Multisim simulation software. Through further analysis and simulation of the improved Sallen-Key filter network of different orders, the Sallen-Key active filter network is designed as the filter forming circuit in the spectrometer amplifier. Through the observation and comparison of the output waveform, the characteristics of the filter forming circuit can be clearly understood and mastered, which provides a reference for the virtual simulation experiment teaching nuclear power electronics.
\end{abstract}

\section{Subject Areas}

Nuclear Technology and Nuclear Instruments

\section{Keywords}

Sallen-Key Filter, Spectrometer Amplifier

\section{Introduction}

In the nuclear electronics experiments, nuclear pulse signal processing system is mainly for nuclear detector output random amplitude and random distribution of nuclear pulse signal processing, through the analysis of nuclear pulse signal detector receives the information can be concluded that the corresponding species, the output signal from the detector is very small, usually the first to sampled signal amplification processing, Although the output signal from the detector by 
the processing of the preamplifier, but as a result of preamplifier magnification and limited due to the narrow pulse peak of nuclear pulse, the pulse signal is bad for the subsequent processing circuit, the signal needs to be spectrometer amplifier amplified to a suitable range, at the same time expand the width of pulse peak convenient and subsequent processing circuit, amplifier, so the spectrometer Different order filters have a very important influence on the pulse signal forming effect [1] [2] [3]. This paper uses Multisim simulation to analyze the effect of different filters and verify the effect of different filters on the circuit performance.

\section{Circuit Parameter Design Calculation}

The integral filter circuit is designed based on the Sallen-key filter. Sallen-key is a topological structure designed for the design of active power filter, with high input impedance and relatively simple structure. Figure 1 is the circuit schematic diagram of Sallen-key filter, which has two-order RC filter. The best SNR is achieved when the waveform is an infinite wide spike pulse, but this waveform cannot be achieved, so the Gaussian pulse waveform after integral filtering has a similar effect. Moreover, the more RC filtering times, the closer the nuclear pulse signal is to the Gaussian filtering [4] [5].

The $A_{u}$ voltage amplification factor of Sallen-key filter is:

$$
A_{u}=\frac{R_{4}+R_{3}}{R_{3}}
$$

$A_{u}$ is the voltage amplification, $R_{3}$ and $R_{4}$ are the resistance values [6].

The voltage transfer function is:

$$
\frac{V_{o}}{V_{i}}=\frac{A}{C_{1} C_{2} R_{1} R_{2} s^{2}+\left(R_{1}\left(C_{1}(1-A)+C_{2}\right)+C_{2} R_{2}\right) s+1}
$$

$A$ is the voltage amplification, $R_{1}$ and $R_{2}$ are the resistance values, $C_{1}$ and $C_{2}$ are capacitance values.

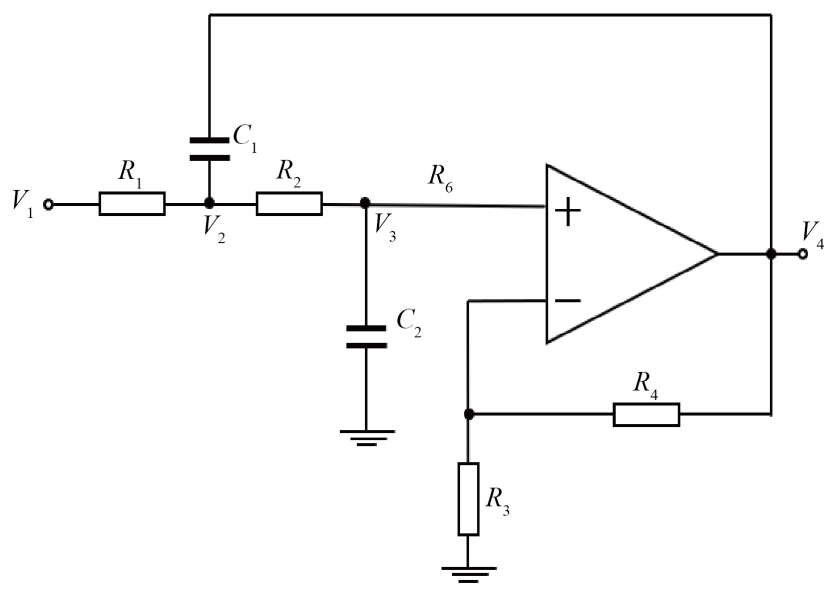

Figure 1. Sallen-Key filter circuit schematic diagram. 
Due to the existence of $C_{1}$, when the input signal frequency is high, the input voltage will be output directly through $C_{1}$ and the amplification factor will be lower than the theoretical calculation. In order to simplify the circuit structure, the circuit can be set as $R_{1}=R_{2}=R, C_{1}=C_{2}=C$. In this case, the voltage transfer function is:

$$
\frac{V_{o}}{V_{i}}=\frac{A}{C^{2} R^{2} s^{2}+(3-A) C R s+1}
$$

$A$ is the voltage amplification.

Because Sallen-key filter is a second-order filter circuit. In order to reflect the influence of different integral parameters on the nuclear pulse signal, a fourth-order Sallen-key filter is used for design. Different parameters are set for sallen-key filters with different orders. The voltage transfer function is:

$$
\begin{aligned}
\frac{V_{o}}{V_{i}} & =\frac{A^{2}}{\left(C_{1}^{2} R^{2} s^{2}+(3-A) C_{1} R s+1\right) \times\left(C_{2}^{2} R^{2} s^{2}+(3-A) C_{2} R s+1\right)} \\
& \times \frac{A^{2}}{\left(C_{3}^{2} R^{2} s^{2}+(3-A) C_{3} R s+1\right) \times\left(C_{4}^{2} R^{2} s^{2}+(3-A) C_{4} R s+1\right)}
\end{aligned}
$$

\section{Simulation of Multisim Circuit}

\subsection{Schematic Design}

The circuit schematic diagram of the spectrometer amplifier is shown in Figure 2. The integral filter circuit can display different filtering effects by selecting different integral filter orders through the switch. Different output modes can be selected by switching SW10 and SW12, and the traffic pass switch SW11 can choose whether to turn on the integral filter circuit [7].

\subsection{Software Emulation}

The initial input signal of the spectrometer amplifier is $10 \mathrm{kHZ}$, and the total width of the square wave pulse with the amplitude of $600 \mathrm{mV}$ is 50 us. The input square wave signal becomes an exponential pulse waveform similar to the nuclear pulse after being amplified by the pole-zero cancellation circuit. After being amplified by the amplifier circuit, the integral filter circuit is input for filtering, and parameter setting is shown in Table 1.

According to the analysis of the simulation results in Figure 3. (A), (B), (C) and (D) correspond to the simulation results of the first-order, second-order, third-order and fourth-order filters. The theoretical magnification power of the first-order Sallen-key filter is 1.5, reaching the theoretical magnification power, but the integral effect after the first-order Sallen-key filter is limited.The theoretical amplification of the second-order Sallen-key filter is 1.5. With the increase of $\mathrm{C}$, the filter circuit has no gain, but the signal has begun to approach the Gaussian pulse, but the rising edge of the signal is still steeper than the falling edge. In order to better improve the integration effect, further integration of 


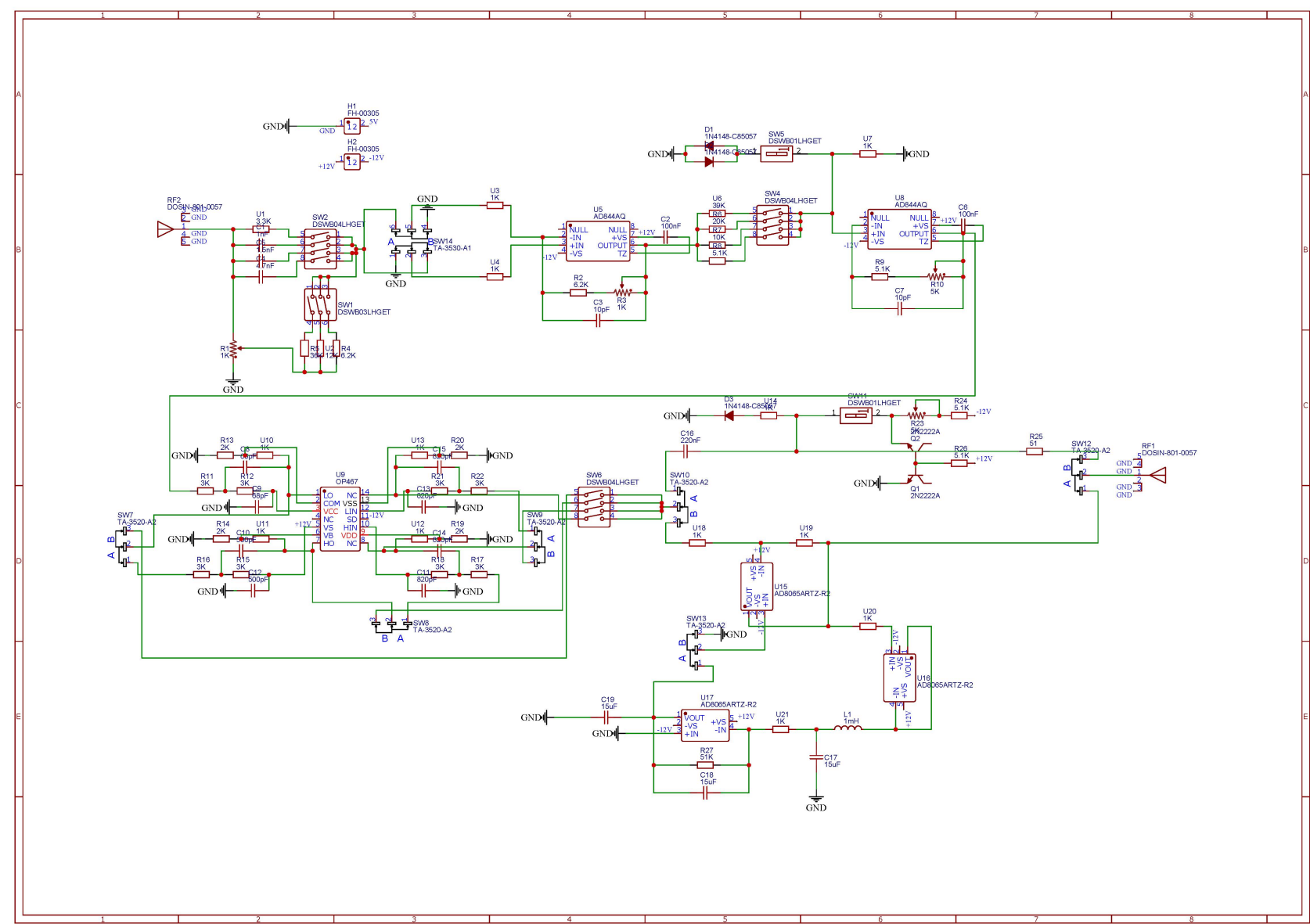

Figure 2. Schematic diagram of spectrometer amplifier.

Table 1. Simulation parameter setting.

\begin{tabular}{ccccccccc}
\hline element & $\mathrm{C}$ & $\mathrm{R} 1$ & $\mathrm{R} 2$ & $\mathrm{R} 3$ & $\mathrm{R} 4$ & Filtering delay & The input peak & The peak output \\
\hline First order & $80 \mathrm{pF}$ & $3 \mathrm{k} \Omega$ & $3 \mathrm{k} \Omega$ & $2 \mathrm{k} \Omega$ & $1 \mathrm{k} \Omega$ & $1 \mathrm{us}$ & $1.091 \mathrm{~V}$ & $1.568 \mathrm{~V}$ \\
Second order & $500 \mathrm{pF}$ & $3 \mathrm{k} \Omega$ & $3 \mathrm{k} \Omega$ & $2 \mathrm{k} \Omega$ & $1 \mathrm{k} \Omega$ & $50 \mathrm{us}$ & $1.568 \mathrm{~V}$ & $1.570 \mathrm{~V}$ \\
Third order & $900 \mathrm{pF}$ & $3 \mathrm{k} \Omega$ & $3 \mathrm{k} \Omega$ & $2 \mathrm{k} \Omega$ & $1 \mathrm{k} \Omega$ & $10 \mathrm{us}$ & $1.570 \mathrm{~V}$ & $1.787 \mathrm{~V}$ \\
Fourth order & $2200 \mathrm{pF}$ & $3 \mathrm{k} \Omega$ & $3 \mathrm{k} \Omega$ & $2 \mathrm{k} \Omega$ & $1 \mathrm{k} \Omega$ & $20 \mathrm{us}$ & $1.787 \mathrm{~V}$ & $1.577 \mathrm{~V}$ \\
\hline
\end{tabular}

the signal is required. The theoretical magnifying power of the third-order Sallen-key filter is 1.5 , and the actual magnifying power is less than the theoretical magnifying power. Compared with the output waveform, the rising edge of the input waveform is slower after the third-order Sallen-key filter, and the waveform is closer to the Gaussian pulse. The theoretical magnifying power of the fourth-order Sallen-key filter is 1.5 , and the actual magnifying power is smaller than the theoretical one. Because $\mathrm{C}$ is too large, the output signal has become smaller. Compared with the output waveform, the waveforms of the rising and falling edges of the model after the fourth-order Sallen-key filter are very similar. The output waveform is very close to the Gaussian pulse waveform, and the final output waveform is very suitable for subsequent signal processing. 


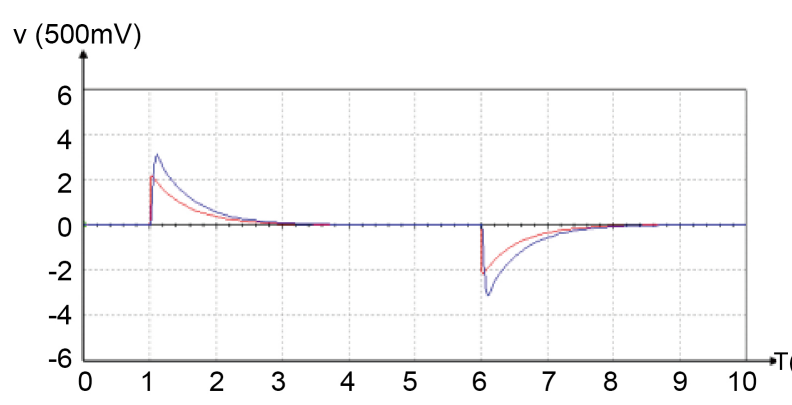

(a)

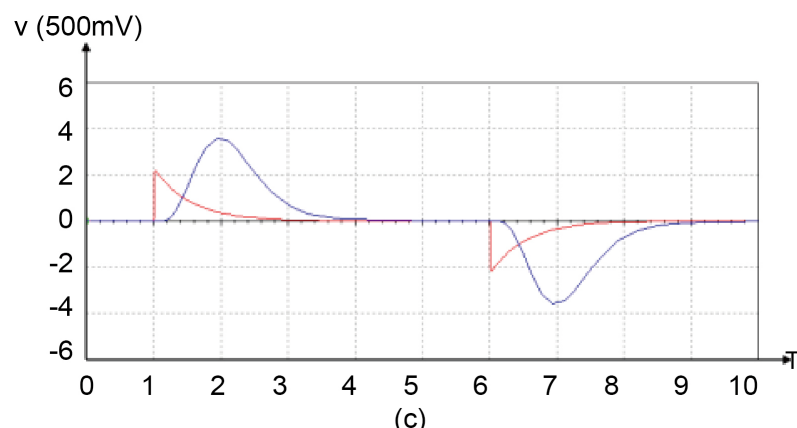

(c)

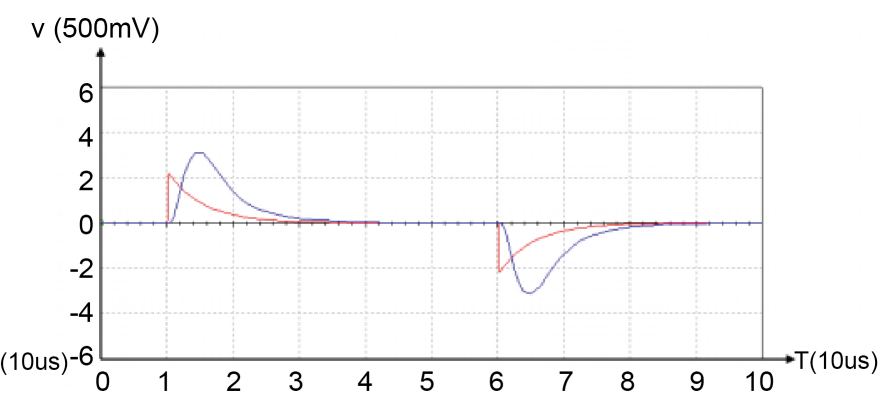

(b)

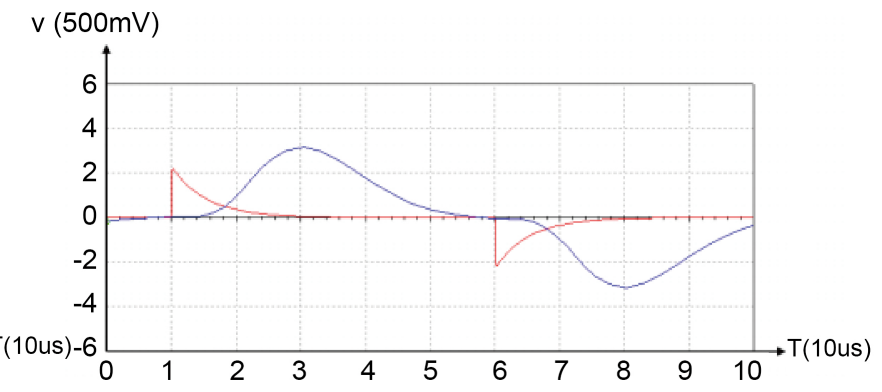

(d)

Figure 3. Output pulse signal Initial pulse signal contrast.

\section{Conclusion}

In the designed spectrometer amplifier, the integral filter circuit is composed of fourth-order Sallen-key filters, and the first to fourth order filters can be freely selected through the switch for output. The simulation results show that the fourth-order Sallen-key filter has excellent quasi-Gaussian output characteristics, which accords with the design of the filter forming circuit of the spectrometer amplifier.

\section{Acknowledgements}

Thanks to the Key Laboratory of Geoscience and nuclear technology of Chengdu University of technology for its equipment support and also for my partners Shi and Jiang for their technical assistance during the experiment.

\section{Conflicts of Interest}

The authors declare no conflicts of interest.

\section{References}

[1] Zha, T. and Ding, Y.X. (2019) Application and Analysis of Integral Filter in LVDT Conditioning Circuit. Journal of Wuhan Polytechnic, 18, 94-98.

[2] Liu, L.Y. and Zhao, X.L. (2013) Application of Multisim10 in Nuclear Electronics Course Design. Science and Technology Information, 26, 165-166.

[3] Cheng, C., Zhao, C.L., Wei, Y.B., et al. (2006) Research on Integrated Amplifier Circuit of Portable Energy Spectrometer. Nuclear Electronics \& Detection Technology, 5, 564-567. 
[4] Zhu, G.P., Yu, X.J., Lu, W.J. (2007) Journal of Electrical and Electronic Teaching, 3, 29-31+34.

[5] Zhang, Z.Y., Zeng, W.H., Zhou, S.M., He, Y.H. and Li, Y.Z. (2011) Design of Pulse Forming Circuit for Nuclear Spectrum Signal Amplifier. Nuclear Electronics \& Detection Technology, 31, 1300-1302.

[6] Hong, Z.L. (2005) Analysis and Design of Analog Integrated Circuit. Science Press, Beijing.

[7] Cheng, F., Cheng, F., Wang, G.-X., et al. (2016) Development of a Nuclear Electronics Training System. College of Nuclear Technology and Automation Engineering, Chengdu University of Technology, Chengdu. 